\title{
Practical Needs in Assessing Response to Therapy in Operated Brain High Grade Glioma According to Response Assessment in Neuro-Oncology Criteria \\ Rania M. Almolla ${ }^{1}$, Hazim I. Tantawy ${ }^{1}$, Mohamed Mahmoud ${ }^{* 1}$, Ahmed A. Morsy ${ }^{2}$, Ahmed A. Bessar ${ }^{1}$ Departments ${ }^{1}$ Radiodiagnosis and ${ }^{2}$ Neurosurgery, Faculty of Medicine, Zagazig University, Egypt. *Corresponding Author: Mohamed Mahmoud, Email: m7amd.m7mod@gmail.com
}

\begin{abstract}
Background: Tumors known as gliomas begin in the brain or spine's glial cells and might spread throughout the body. An effort was made to improve tumor response evaluation and end point selection through the Response Assessment in Neuro-Oncology (RANO) working group. Objective: To standardize the assessment method and results reporting by applying MRI-RANO criteria in detecting glioma response to surgical treatment.

Patients and Methods: This was a prospective cohort study included 12 patients ( 4 males and 8 females) whose mean age was $53.6 \pm 15$.1years. Included patients are those who were operated and pathologically proved as brain glioma grade IV (GBM). They underwent post-operative MRI within 48 hours and are available for follow up MRI after 3 months. Imaging modalities applied in both MRI exams were conventional magnetic resonance imaging, MRI diffusion weighted images, and contrast imaging. RANO criteria were applied.

Results: According to RANO criteria, none of the lesions had pseudo response or pseudo progression. However, two thirds $(66.7 \%)$ had progressive disease and one third (33.3\%) had complete response. There was statistically significant association between MRI findings 3 months postoperative and RANO criteria as patients with compete response had statistically significant decrease on measurable solid components, diffusion restriction, post contrast enhancement and all lesions had grade I edema with no mass effect or midline shift compared to patients with progressive disease. 4 cases that showed complete response category had fulfilled all the RANO criteria, while 8 cases with category progressive disease had fulfilled more than one of the described RANO criteria.

Conclusion: RANO criteria are an effective tool to be used in interpretation of MRI for follow-up of surgically operated glioma patients to detect their response.
\end{abstract}

Keywords: Therapy response, Brain high grade glioma, Neuro-oncology criteria.

\section{INTRODUCTION}

Brain or spinal cord tumors known as gliomas arise from the glial cells that surround and support the brain or spine, respectively. Gliomas account for around thirty percent of all brain tumors and almost eighty percent of all malignant brain tumors ${ }^{(1)}$. Gliomas are the most frequent type of brain cancer, and new treatment modalities have necessitated the development of accurate tools for evaluating the disease's response. Surgery, radiation, and/or new chemotherapy drugs are currently used to treat high-grade gliomas, however there are additional options ${ }^{(2)}$.

It was decided to create the Response Evaluation in Neuro-Oncology (RANO) working group in order to enhance tumor response assessment and end goal selection. Endpoints and criteria for determining therapy response have evolved due to advancements in imaging technologies, in particular ${ }^{(3)}$. In patients with high-grade glioma, the MacDonald criteria were proposed in 1990 as the gold standard for evaluating response and progression. Failure to provide guidance on pseudoprogression, pseudo-response, and tumor progression while using this response criterion posed a challenge. The MacDonald criteria only included illness progression that enhanced contrast and ignored disease progression that did not enhance contrast ${ }^{(4,5)}$. For the RANO criteria, progression is defined as an increasing level of nonenhancing illness. The RANO criteria argue that any qualitative increase in non-enhancing disease represents progression as it could be difficult to quantify non- enhancing disease progression ${ }^{\left({ }^{(}\right)}$. It was found that the Macdonald criteria had three key flaws that were corrected by the RANO guidelines: Standardizing imaging definitions, such as the size and number of enhanced T1 lesions that are measurable and nonmeasurable, pseudo-progression and pseudo-response interpretation for changes in $\mathrm{T} 2$ and FLAIR signal abnormalities to be taken into consideration when evaluating the impact of antiangiogenic treatments, and widening the radiographic response ${ }^{(7)}$. Shorter trial durations and lower drug development costs can be achieved with endpoints based on imaging that include tumor evaluation and measurement. By distinguishing between therapy response and tumor development, radiologists can help patients avoid harmful treatment changes ${ }^{(2)}$.

We aimed at this study to standardize the assessment method and results reporting by applying MRI-RANO criteria in assessment of glioma response to surgical treatment.

\section{PATIENTS AND METHODS}

A Prospective cohort study was carried out at the Radiodiagnosis Department-Zagazig University Hospitals. All the patients were referred from Neurosurgery Department of Zagazig University Hospitals from January 2021 to June 2021.

Ethical approval:

The study was approved by Zagazig University' Research Ethics Committee, (ZU-IRB\#6210/25-92020). This work has been carried out in accordance 
with The Code of Ethics of the World Medical Association (Declaration of Helsinki) for studies involving humans. Every participant signed an informed consent form to share in the study.

Those who were operated and pathologically proved to be brain glioma grade IV (GBM) were 12 patients (4 males and 8 females) and their ages ranged from 25 to 69 years $(53.6 \pm 15.1$ years).

Inclusion criteria: Patients who underwent postoperative MRI within 48 hours and are available for follow up MRI after 3 months.

Exclusion criteria: Patients who cannot undergo an MRI because of contraindications such as pacemakers or other cardiac devices put in their hearts, ocular implants, aneurysmal clips, or claustrophobia, a significant time had passed since these patients had surgery, thus there aren't any immediate post-operative MRI images accessible, and missed patients during follow up.

All patients were subjected to the following:

I. General examination:

-Personal history: Name, age, occupation.

-Present history (complaints): Headache, nausea, gait disturbance, vomiting, visual disturbance, and lateralization.

II. Neurological examination: Carried out by our colleagues in Neurosurgery Department. All patients were diagnosed with glioma according to $\mathrm{CT}$ and cMRI findings and surgically treated then referred them to do postoperative MRI assessment within 48 hours and followed up by another MRI examination 3 months later then application of RANO criteria to assess outcome.

IV. Histopathological examination: Surgical complete resection of tumors done for all 12 patients. Histopathology was done after operation to be correlated with MRI results.

III. Imaging modalities: Conventional magnetic resonance imaging (cMRI), MRI diffusion weighted images (DWI), contrast imaging.

Imaging Modalities: After 48 hours of operation:

A) Conventional MRI Images (cMRI):

- cMRI was used in all cases, with pictures produced by

1.5-Tesla superconducting MR images.

- All cases were checked with a conventional head coil while lying supine.

- Axial, coronal, and sagittal views were obtained of different pulse sequences.

The following protocol was used to investigate each case:

Sagittal T1 WI as a localizer: $\mathrm{TE}=10-12 \mathrm{~ms}$, TR $=$ 400-600 ms. Axial, sagittal and coronal spin-echo sequences, short TR/TE (T1-weighted images): TE = $10-12 \mathrm{~ms}, \mathrm{TR}=400-600 \mathrm{~ms}$. Axial, sagittal and coronal fast spin-echo, long TR/TE (T2-weighted images): $\mathrm{TE}=70-90 \mathrm{~ms}, \mathrm{TR}=2800-3500 \mathrm{~ms}, \mathrm{TI}=$ 2000 ms. Axial FLAIR: TE $=302 \mathrm{~ms}$, TR $=4800 \mathrm{~ms}$, $\mathrm{TI}=1650 \mathrm{~ms}$.

\section{B) Diffusion weighted MRI Images (DW-MRI):}

Multisectoral single shot spin echo EPI sequence was used for the DWI imaging parameter, and the slice thickness ranged from $4500 / 120$ to 140 (TR/TE) (5 $\mathrm{mm})$. We used a sequence of echo-planar imaging (EPI) to obtain the DWIs $(0,500,1000 \mathrm{~s} / \mathrm{mm} 2 \mathrm{~b}$ values $)$ while suppressing fat with a frequency-selective radio frequency (RF) pulse prior to the pulse sequence. Maps and values were generated on a computer by applying numerous ROI (regions of interest) on the lesion, and the lowest value was recorded as an ADC measurement result. The ADC values were expressed in $10^{-3} \mathrm{~mm}^{2} / \mathrm{sec}$.

C- Post contrast axial, sagittal and coronal spin-echo sequences, short TR/TE (T1- weighted images): TE $=10-12 \mathrm{~ms}, \mathrm{TR}=400-600 \mathrm{~ms}$ Omniscan or Magnivist [Gadolinium diethelene triamine penta acetic acid ("GdDTPA") $0.1 \mathrm{mmol} / \mathrm{kg}$ body weight were used to administer contrast material intravenously, and a contrast enhanced T1 Weighted spin echo sequence was acquired in the axial, coronal and sagittal planes.

Follow up MRI was done after 3 months from the operation where the same MRI protocol done at 48 hours examination was applied.

\section{Image analysis:}

We assessed the post operative signal changes at the operative bed regarding hemorrhagic signal, ischemia areas and presence of abnormal enhancement (according to site and size of contrast enhancementDWI, FLAIR, T2 and T1 character). Treatment plan and clinical response to treatment was assessed through direct contact with patient's clinician. After three months, the same MRI procedure used for the 48-hour assessment was repeated to check for measurable elevated solid components, encephalomalacia, and gliosis. Results from RANO criteria and any pseudoprogression or pseudo-responses were evaluated in relation to treatment category.

Table (1): RANO criteria

\begin{tabular}{|l|c|c|c|c|}
\hline Criterion & CR & PR & SD & PD \\
\hline T1-Gd + & None & $\geq 50 \% \downarrow$ & $\begin{array}{c}<50 \% \downarrow \text { to } \\
<25 \% \uparrow\end{array}$ & $\begin{array}{c}\geq 25 \% \\
\uparrow\end{array}$ \\
\hline T2/FLAIR & $\begin{array}{c}\text { Stable } \\
\text { or } \downarrow\end{array}$ & $\begin{array}{c}\text { Stable } \\
\text { or } \downarrow\end{array}$ & $\begin{array}{c}\text { Stable or } \\
\downarrow\end{array}$ & $\uparrow$ \\
\hline New lesion & None & None & None & Present \\
\hline Corticosteroids & None & $\begin{array}{c}\text { Stable } \\
\text { or } \downarrow\end{array}$ & $\begin{array}{c}\text { Stable or } \\
\downarrow\end{array}$ & Na \\
\hline Clinical status & $\begin{array}{c}\text { Stable } \\
\text { or } \uparrow\end{array}$ & $\begin{array}{c}\text { Stable } \\
\text { or } \uparrow\end{array}$ & $\begin{array}{c}\text { Stable or } \\
\uparrow\end{array}$ & $\downarrow$ \\
\hline $\begin{array}{l}\text { Requirement } \\
\text { for response }\end{array}$ & All & All & All & Any \\
\hline
\end{tabular}

$-\mathrm{CR}=$ complete response. $\quad-\mathrm{PR}=$ partial response. stationary disease. $-\mathrm{PD}=$ progressive disease.

\section{Statistical analysis}

Researchers used SPSS (statistical tool for social science, version 18). After the data were gathered and analysed, it was presented in table and diagram formats. The mean, standard deviation, range, frequency, and 
percentage were all computed as descriptive statistics. The following tests were used: Students' t test, Pearson's product correlation coefficient, and the Chi-Square $\left(\mathrm{X}^{2}\right)$ test. A $\mathrm{p}$ value was found to be significant using the following criteria: The difference is not statistically significant if $\mathrm{P}$ is greater than 0.05 ; however, if $\mathrm{P}$ is equal to or less than 0.05 , the difference is significant.

\section{RESULTS}

The study included 12 patients (4 males and 8 females) their ages ranged from 25 years to 69 (53.6 \pm 15.1years). All studied group (100.0\%) received combined chemo- and radio-therapy following the operation.

Table (2) showed the MRI findings at 48 hours and 3 months post-operative where there was statistically significant reduction in edema, mass effect, T2 and FLAIR signals 3 months postoperatively. Post-operative hemorrhagic signal was reduced in follow up after 3 months but with no significant difference. Marginal enhancement in the immediate post-operative was detected in all cases, however, in the 3 months follow up it was limited to 10 cases and only 8 cases of them showed recurrent solid measurable component. DWI showed that marginal restriction was the finding in the immediate $48 \mathrm{~h}$ in the 8 cases, which mostly is related to surgical interference ischemia. However, in the 3 months follow up, 6 of the measurable enhanced lesions displayed diffusion restriction and two of the measurable recurrent masses hadn't definite restriction.

Table (3) showed that according to RANO criteria, none of the lesions had pseudo response or pseudo progression. However, two thirds $(66.7 \%)$ had progressive disease and one third (33.3\%) had complete response.

Table (4) showed that there was statistically significant association between MRI findings 3 months postoperative and RANO criteria as patients with complete response had statistically significant decrease on measurable solid components, diffusion restriction, post-contrast enhancement and all lesions had grade I edema with no mass effect or midline shift compared to patient with progressive disease.

Table (5) showed that 4 cases with complete response category had fulfilled all the RANO criteria, while 8 cases with category progressive disease had fulfilled more than one of the described RANO criteria.

Table (6) showed that older age patients had a significant increase in progressive course than younger age. Regarding patients sex and pre-operative lesion size, there was no statistically significant difference.

Table (2): Comparing the MRI descriptive criteria of the lesions 48 hours and 3 months post-operative by MRI

\begin{tabular}{|c|c|c|c|}
\hline & $\begin{array}{c}48 \text { hours } \\
\text { post-operative }\end{array}$ & $\begin{array}{c}3 \text { months } \\
\text { post-operative }\end{array}$ & $P_{\text {_Value }}$ \\
\hline ( & No(\%) & No(\%) & \\
\hline $\begin{array}{l}\text { - Grade I edema with no mass affect } \\
\& \text { midline shift }\end{array}$ & $2(16.7 \%)$ & $6(50.0 \%)$ & \\
\hline $\begin{array}{l}\text { - Grade II edema with no mass } \\
\text { affect \& midline shift }\end{array}$ & $6(50.0 \%)$ & $2(16.7 \%)$ & $0.03^{*}$ \\
\hline $\begin{array}{l}\text { - Grade II edema with mass affect \& } \\
\text { midline shift }\end{array}$ & $0(0.0 \%)$ & $2(16.7 \%)$ & \\
\hline $\begin{array}{l}\text {-Grade III edema with mass affect \& } \\
\text { midline shift }\end{array}$ & $4(33.3 \%)$ & $2(16.7 \%)$ & \\
\hline $\begin{array}{l}\text { T1 } \\
\text { - Hemorrhage } \\
\text { - No hemorrhage }\end{array}$ & $\begin{array}{l}6(50.0 \%) \\
6(50.0 \%)\end{array}$ & $\begin{array}{l}4(33.3 \%) \\
8(66.7 \%)\end{array}$ & 0.6 \\
\hline $\begin{array}{l}\text { FLAIR } \\
\text { - Iso intensity } \\
\text { - Low intensity } \\
\text { - High intensity } \\
\text { - Both low \& high }\end{array}$ & $\begin{array}{c}0(0.0 \%) \\
0(0.0 \%) \\
12(100 \%) \\
0(0.0 \%)\end{array}$ & $\begin{array}{l}0(0.0 \%) \\
4(33.3 \%) \\
6(50.0 \%) \\
2(16.7 \%)\end{array}$ & $0.01^{*}$ \\
\hline $\begin{array}{l}\text { DWI } \\
\text { - Restriction } \\
\text { - No restriction } \\
\end{array}$ & $\begin{array}{l}8(66.7 \%) \\
4(33.3 \%) \\
\end{array}$ & $\begin{array}{l}6(50.0 \%) \\
6(50.0 \%) \\
\end{array}$ & 0.4 \\
\hline \begin{tabular}{l}
\multicolumn{1}{c}{ Contrast } \\
-Marginal enhancement \\
-Enhanced solid measurable lesion \\
-No enhancement
\end{tabular} & $\begin{array}{l}12(100 \%) \\
(0.0 \%) \\
0(0.0 \%)\end{array}$ & $\begin{array}{l}10(83.3 \%) \\
8(66.7 \%) \\
2(16.7 \%)\end{array}$ & 0.4 \\
\hline
\end{tabular}


Table (3): Response to therapy according to RANO criteria in the 3 months follow up of the lesions among the studied group

\begin{tabular}{|l|c|c|}
\hline \multicolumn{1}{|c|}{ RANO } & \multicolumn{2}{c|}{$\begin{array}{c}\text { The studied group } \\
\text { No - 12 }\end{array}$} \\
\hline & No & $\%$ \\
\hline * Pseudo response & 12 & $100.0 \%$ \\
- Negative & 12 & $100.0 \%$ \\
\hline * Pseudo progression & & $33.3 \%$ \\
- Negative & 4 & $66.7 \%$ \\
\hline * Category & 8 & - Complete response \\
- Progressive disease & & \\
\hline
\end{tabular}

Table (4): Relation between RANO criteria and MRI characteristics of the lesion after 3 months follow up

\begin{tabular}{|c|c|c|c|}
\hline Variables & $\begin{array}{l}\text { Complete response } \\
\text { No }=4\end{array}$ & $\begin{array}{c}\text { Progressive disease } \\
\text { No }=8\end{array}$ & P_Value \\
\hline $\begin{array}{l}\text { Solid Components } \\
\text { - Measurable } \\
\text { - Non Measurable }\end{array}$ & $\begin{array}{c}0(0.0 \%) \\
4(100.0 \%)\end{array}$ & $\begin{array}{c}8(100.0 \%) \\
0(0.0 \%)\end{array}$ & $0.02^{*}$ \\
\hline $\begin{array}{l}\text { Contrast } \\
\text { - Enhancement } \\
\text { - No-enhancement }\end{array}$ & $\begin{array}{c}2(50.0 \%),(\text { thin marginal }) \\
2(50.0 \%)\end{array}$ & $\begin{array}{c}8(100.0 \%) \\
0(0.0 \%)\end{array}$ & $0.02^{*}$ \\
\hline $\begin{array}{l}\text { DWI } \\
\text { - Restriction } \\
\text { - No restriction }\end{array}$ & $\begin{array}{c}0(0.0 \%) \\
4(100.0 \%)\end{array}$ & $\begin{array}{l}6(75.0 \%) \\
2(25.0 \%)\end{array}$ & $0.01^{*}$ \\
\hline $\begin{array}{l}\text { T2 } \\
\text { Grade I } \\
\text { Grade II } \\
\text { Grade III } \\
\text { Grade IV }\end{array}$ & $\begin{array}{c}4(100.0 \%) \\
0(0.0 \%) \\
0(0.0 \%) \\
0(0.0 \%)\end{array}$ & $\begin{array}{l}2(25.0 \%) \\
2(25.0 \%) \\
2(25.0 \%) \\
2(25.0 \%)\end{array}$ & $0.03^{*}$ \\
\hline $\begin{array}{l}\text { FLAIR } \\
\text { - Low intensity } \\
\text { - High intensity } \\
\text { - Both low \& high }\end{array}$ & $\begin{array}{c}4(100.0 \%) \\
0(0.0 \%) \\
0(0.0 \%) \\
\end{array}$ & $\begin{array}{l}0(0.0 \%) \\
6(75.0 \%) \\
2(25.0 \%) \\
\end{array}$ & $0.02^{*}$ \\
\hline $\begin{array}{l}T_{1} \\
\text { - Hemorrhage } \\
\text { - No hemorrhage }\end{array}$ & $\begin{array}{c}0(0.0 \%) \\
4(100.0 \%)\end{array}$ & $\begin{array}{l}4(50.0 \%) \\
4(50.0 \%)\end{array}$ & 0.08 \\
\hline
\end{tabular}

Table (5): RANO criteria summarization for our cases

\begin{tabular}{|l|c|c|c|c|c|c|}
\hline No. & T1-Gd & T2-FLAIR & New lesion & corticosteroid & $\begin{array}{c}\text { Clinical } \\
\text { status }\end{array}$ & Response \\
\hline $\mathbf{1}$ & $+\geq 25 \%$ & $\uparrow$ & + & Increased & Declined & PD \\
\hline $\mathbf{2}$ & None & Stable & - & None & Stable & CR \\
\hline $\mathbf{3}$ & $+\geq 25 \%$ & $\uparrow$ & + & Increased & Declined & PD \\
\hline $\mathbf{4}$ & $+\geq 25 \%$ & $\uparrow$ & + & Increased & Declined & PD \\
\hline $\mathbf{5}$ & $+\geq 25 \%$ & Stable & + & Increased & Declined & PD \\
\hline $\mathbf{6}$ & None & $\downarrow$ & - & None & Improved & CR \\
\hline $\mathbf{7}$ & None & $\downarrow$ & - & None & Improved & CR \\
\hline $\mathbf{8}$ & $+\geq 25 \%$ & $\uparrow$ & + & Increased & Declined & PD \\
\hline $\mathbf{9}$ & $+\geq 25 \%$ & Stable & + & Increased & Declined & PD \\
\hline $\mathbf{1 0}$ & $+\geq 25 \%$ & $\uparrow$ & + & Increased & Declined & PD \\
\hline $\mathbf{1 1}$ & None & Stable & - & None & Stable & CR \\
\hline $\mathbf{1 2}$ & $+\geq 25 \%$ & $\uparrow$ & + & Increased & Declined & PD \\
\hline
\end{tabular}


Table (6): Relation between patients' age, sex and lesion size and RANO criteria

\begin{tabular}{|c|c|c|c|}
\hline Variables & $\begin{array}{c}\text { Complete response } \\
\text { No }=4\end{array}$ & $\begin{array}{c}\text { Progressive disease } \\
\text { No }=8\end{array}$ & P-Value \\
\hline $\begin{array}{l}\text { Age (years) } \\
\text { Mean } \pm \text { SD }\end{array}$ & $41.3 \pm 10.7$ & $62.4 \pm 4.7$ & $0.03^{*}$ \\
\hline $\begin{array}{l}\text { Lesion size preoperative } \\
\text { Mean } \pm \text { SD } \\
\text { Median } \\
\end{array}$ & $\begin{array}{c}34.5 \pm 5.8 \\
34.5 \\
\end{array}$ & $\begin{array}{c}42.4 \pm 20.3 \\
37\end{array}$ & 0.6 \\
\hline \begin{tabular}{|l|} 
Sex \\
Male \\
Female \\
\end{tabular} & $\begin{array}{c}0(0.0 \%) \\
4(100.0 \%)\end{array}$ & $\begin{array}{l}4(50.0 \%) \\
4(50.0 \%)\end{array}$ & 0.08 \\
\hline
\end{tabular}

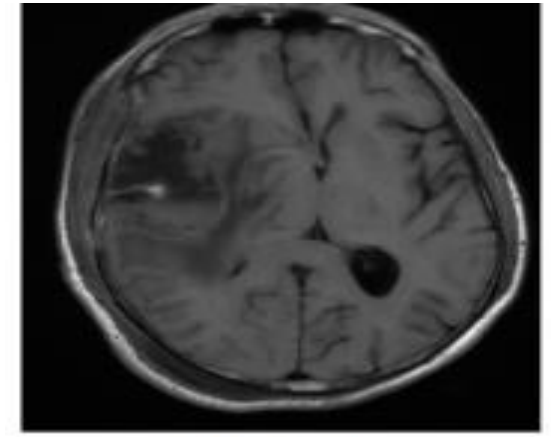

A: axial TIWI
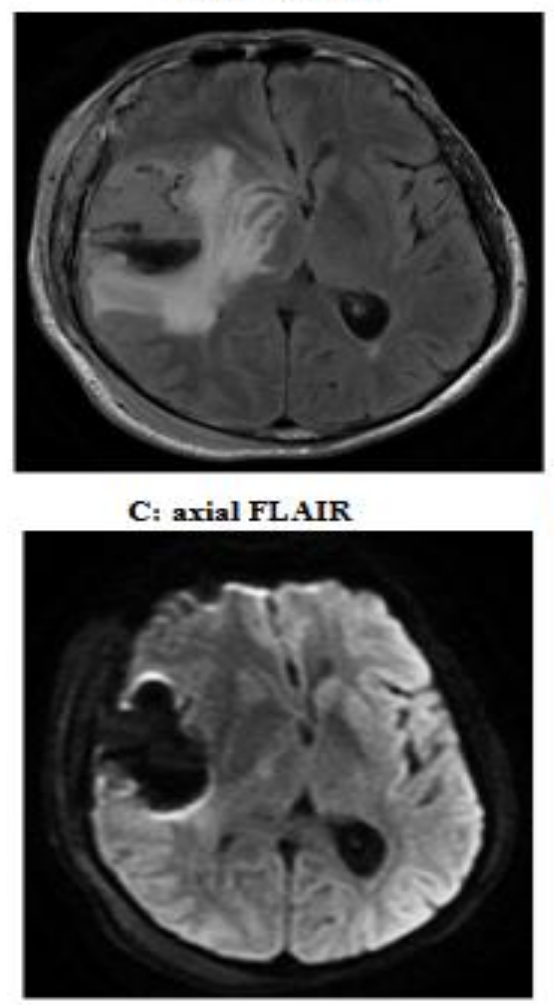

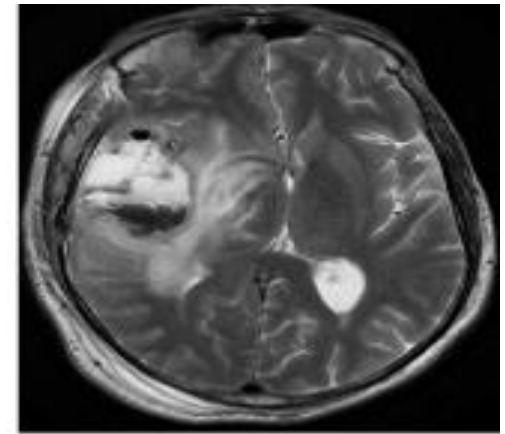

B: axial T2WI

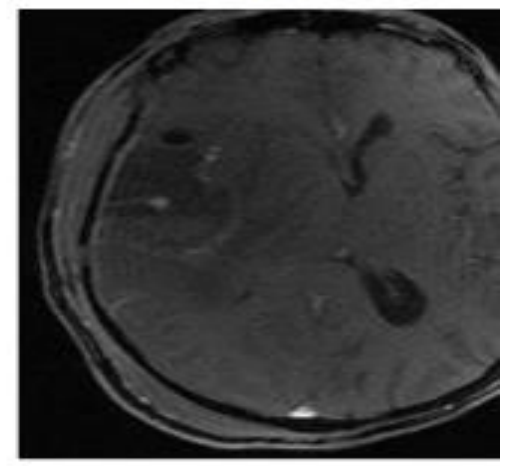

D: axial TIWI post contrast

\section{E: axial DWI}

Figure (1): 48 hours post-operative MRI of Right temporo-pareital pathology proved high grade glioma (GBM). A 59years-old male patient presented by left sided weakness. A: Axial T1WI: Cystic lesion with fluid/fluid level, containing hemorrhagic element of early subacute stage (high signal on T1 and corresponding low signal on T2WIs image B). B: Axial T2WI showing perilesional edema grade II with mass effect effacing the RT lateral ventricle and related cortical sulci with midline shift to the left. C: Axial FLAIR grade III cytotoxic edematous changes were noted causing mass effect on right lateral ventricle and related cortical sulci with midline shift to the left. D: Axial TIWI with contrast (gadolinium DTPA): Marginal thin enhancement was noted. E: Axial DWI (b 1000): Marginal restriction was demonstrated. 


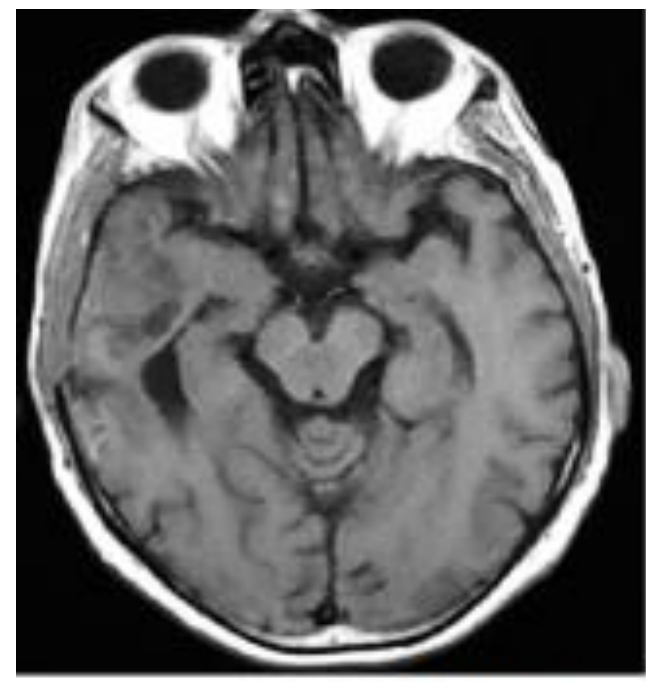

A: axial TIVI

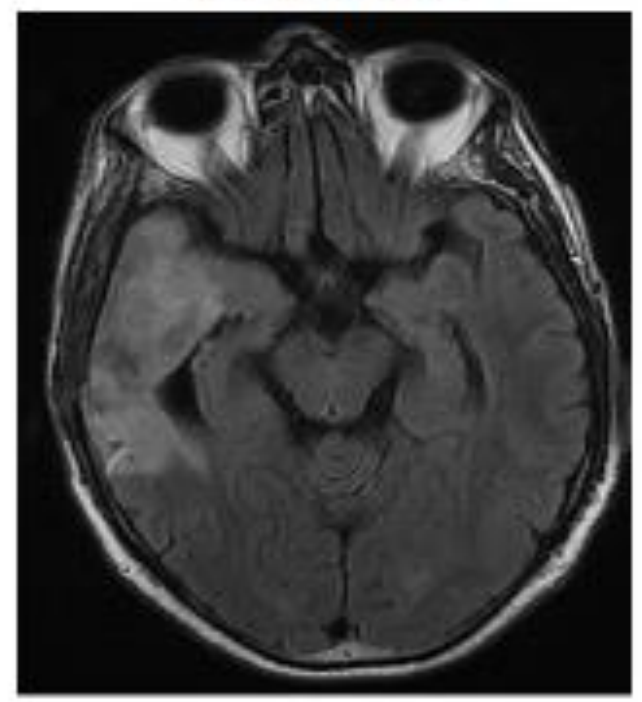

C: axial FLAIR

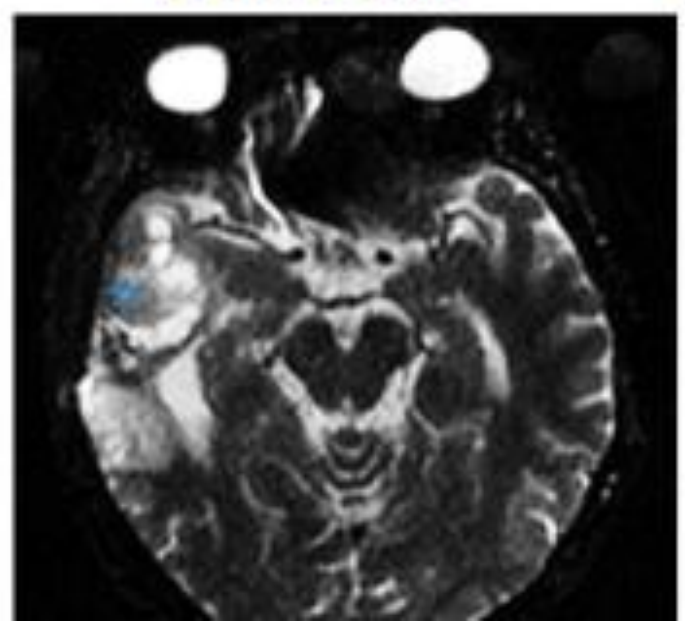

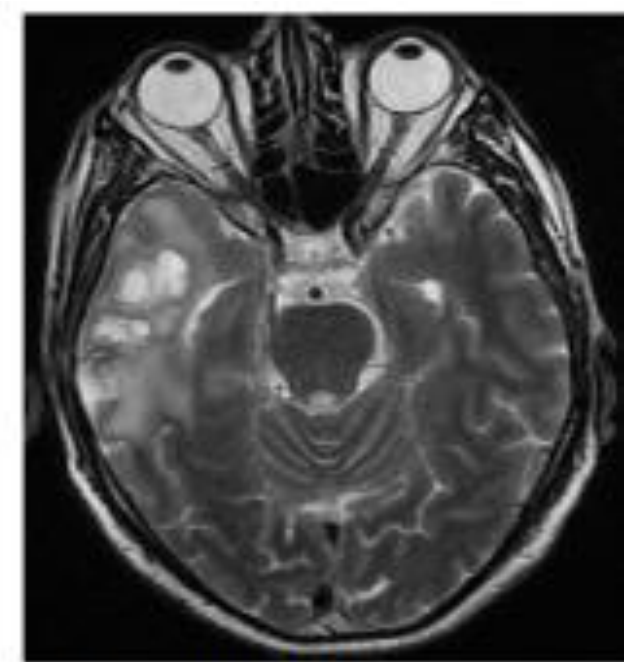

B: axial T2WI

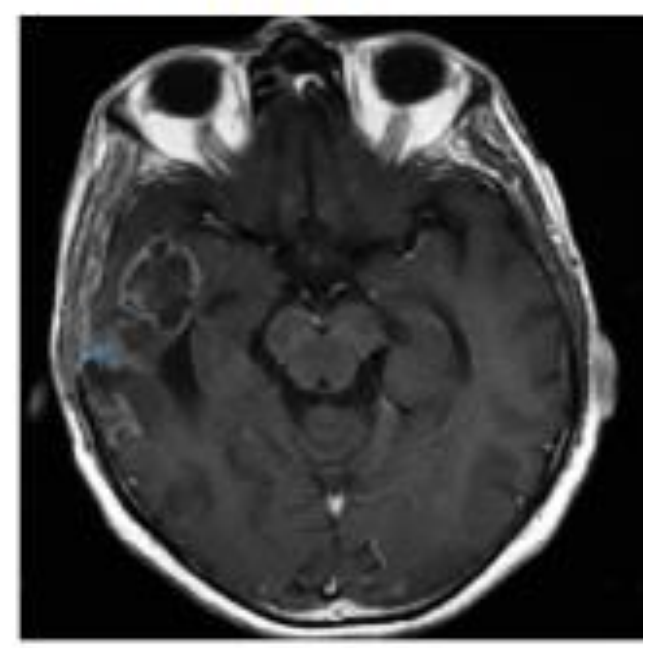

D: axial TIWI post contrast

\section{E: axial DWI}

Figure (2): 3 months postoperative follow up MRI for same patient A: Axial T1WI where localized area of marginal and gyral hyperintensities was noted mostly residual hemorrhagic component B: Axial T2WI: Heterogeneous high signal with perilesional edema grade I. C: Axial FLAIR: Heterogeneous high signal with increased FLAIR hyperintensities compared to 48h. D: Axial TIWI with contrast (gadolinium DTPA): Marginally enhanced cavitary lesion with newly enhanced measurable solid component $23 \times 17 \mathrm{~mm}$ is depicted. E: Axial DWI (b 1000): Multiple areas of restricted diffusion within the solid components were seen. Solid component: measurable solid component ( $23 \mathrm{x} 17 \mathrm{~mm})$ was seen. RANO category: progressive course (PR). 


\section{DISCUSSION}

Primary central nervous system cancers such as gliomas are extremely frequent. Grade I/II low-grade astrocytomas, anaplastic astrocytomas, and glioblastoma multiforme are the three types of these tumors (grade IV) (8). When treating a glioma patient, imaging is crucial in all phases of care, from early detection to long-term monitoring, a thorough understanding of tumor biology and the unique alterations expected as a result of each treatment method is required for interpreting results after surgery, radiation, and chemotherapy ${ }^{(9)}$. This criterion takes into account non-enhancing lesions via T2/FLAIR imaging when evaluating response and includes specific guidance to assist reviewers in differentiating between pseudo progression and true $\mathrm{PD}^{(3)}$.

In our study, immediate MRI was done 48 hours postoperative as a base line to: (1) Confirm if there was residual sizable masses and hemorrhagic changes, (2) Detect DWI changes (post-surgical ischemic marginal restriction or residual restricted masses) and (3) Detect residual enhanced masses ${ }^{(10)}$. According to RANO criteria FLAIR and T2 sequences were analysed. On post-operative MRI, $100 \%$ of cases (12 cases) displayed high signal on FLAIR, which persisted only in progressive disease ( 8 cases) in follow up after 3 months. T2 sequence showed redistribution of the perilesional edematous changes with $50 \%$ of cases that showed grade I edema in 3 months follow up series. This is in agreement with Xiao Shen et al. ${ }^{(10)}$.

RANO criteria also included the patient clinical status as an indicator of disease response to therapy. in our study, 8 cases out of $12(66.7 \%)$ showed clinical decline that was corresponding to recurrent solid lesions while the other 4 patients (33.3\%) showed good clinical status either stable or even improved (50:50\%). This is matching with another study, which reported a clinical decline due to progressive course in 63.6 percent of their patients ${ }^{(11)}$.

In our study according to RANO criteria, 4 cases $(33.3 \%)$ showed complete response while 8 cases $(66.7 \%)$ showed progressive disease. This disagrees with another study by Huang ${ }^{(12)}$ where 34 cases $(65.3 \%$ ) had complete responses and 8 cases $(34.7 \%)$ had progressing disease. This could be owing to differences in selection process or treatment protocols. Nevertheless, there was no evidence of a partial response or a stable illness outcome in either of the investigations. Different studies agree with our study. A study evaluated 13 patients found that 4 cases showed complete response to treatment $(30.7 \%)^{(13)}$. Another one reported 7 cases $(63.6 \%)$ out of 11 patients included in their study showed progressive course as we noticed ${ }^{(11)}$. These studies included nearly the same numbers of the patients in our study, which intensify our results. We agree with Al Sayyari ${ }^{(14)}$ and Alexiou et al. ${ }^{(15)}$ who evaluated 16 and 30 patients of GBM respectively, to assess presence of recurrent masses in duration of 15-16 weeks after operation. They found PD in $56.25 \%$ and $53.3 \% \%$ and complete response in $31.25 \%$ and $20 \%$ respectively. No cases showed pseudo-progression, however they reported pseudoresponse in 2 and 8 cases respectively. Their detection of pseudo response may be due to different treatment lines. Different studies agree with our results regarding ratio of cases with complete response where Baek et al. ${ }^{(16)}$ and Chung et al. ${ }^{\left({ }^{(17)}\right.}$ reported $34.1 \%$, while D'Souza et al. (18) reported $37 \%$.

Regarding progressive courses, Kim et al. ${ }^{(19)}$ reported $61.1 \%$ and Di Constanzo et al. ${ }^{(20)}$ reported $58.6 \%$ that nearly agree with our study that showed $67.7 \%$ progressive disease. Other studies disagree with ours either due to selection bias or different treatment plan for example regarding complete response a study by Cha et al. ${ }^{(19)}$ showed $57.14 \%$ and one by Sundgren $\boldsymbol{e t}$ al. ${ }^{(21)}$ showed $47 \%$, which disagree with our study that showed $33.3 \%$ of complete response.

\section{CONCLUSION}

RANO criteria are an effective tool to be used in interpretation of MRI for follow-up of surgically operated glioma patients to detect their response.

\section{Financial support and sponsorship: Nil. Conflict of interest: Nil.}

\section{REFERENCES}

1. Mamelak A, Jacoby D (2007): Targeted Delivery of Antitumoral Therapy to Glioma and Other Malignancies with Synthetic Chlorotoxin (TM-601). Expert Opin Drug Deliv., 4 (2): 175-186.

2. Kessler A, Bhatt A (2018): Brain tumor post-treatment imaging and treatment related complications. Insights Imaging, (6): 1057-1075.

3. Wen P, Macdonald D, Reardon D et al. (2010): Updated response assessment criteria for high-grade gliomas: Response assessment in neuro-oncology working group. J Clin Oncol., 28: 1963-1972.

4. Macdonald D, Cascino T, Schold S et al. (1990): Response criteria for phase II studies of supratentorial malignant glioma. J Clin Oncol., 8: 1277-1280.

5. Eisele $\mathbf{S}$, Wen $\mathbf{P}$, Lee $\mathbf{E}$ (2016): Assessment of brain tumor response: RANO and its offspring. Curr Treat Options Oncol., 17 (7): 35-39.

6. Chukwueke U, Wen $P$ (2019): Use of the Response Assessment in Neuro-Oncology (RANO) criteria in clinical trials and clinical practice. CNS Oncol., 8 (1): 28-43.

7. Yang D (2016): Standardized MRI assessment of high-grade glioma response: a review of the essential elements and pitfalls of the RANO criteria. Neuro Oncology Practice, 3 (1): 59-67.

8. Jeuna S, Kima M, Kim B et al. (2005): Assessment of malignancy in gliomas by $3 \mathrm{~T} 1 \mathrm{H}$ MR spectroscopy. Clin Imaging, 29: 10-15.

9. Leao D, Craig P, Godoy L et al. (2020): Response Assessment in Neuro-Oncology Criteria for Gliomas: Practical Approach Using Conventional and Advanced Techniques. Am J Neuroradiol., 41: 10-20.

10. Shen X, Wang E, Yao C et al. (2017): Application of magnetic resonance spectroscopy in the preoperative grading of gliomas: International Journal Clin Exp., 10 (2): 2834-2841.

11. Bolcaen J, Acou M, Boterberg T et al. (2017): 18F-FCho PET and MRI for the prediction of response in glioblastoma patients according to the RANO criteria. Nuclear Medicine Communications, 38 (3): 242-249.

12. Huang $R$, Rahman $R$, Ballman $K$ et al. (2015): The Impact of T2/FLAIR Evaluation per RANO Criteria on Response 
Assessment of Recurrent Glioblastoma Patients Treated with Bevacizumab. Clinical Cancer Research, 22 (3): 575-581.

13. Ellingson B, Wen P, Timothy $\mathbf{F}$ et al. (2017): Modified Criteria for Radiographic Response Assessment in Glioblastoma Clinical Trials. Neurotherapeutics, 14: 307-320.

14. Al Sayyari A, Buckley R, McHenery C et al. (2010): Distinguishing recurrent primary brain tumor fromadiation injury: a preliminary study using a susceptibility-weighted MR imaging guided apparent diffusion coefficient analysis strategy. AJNR Am J Neuroradiol., 31: 1049-105.

15. Alexiou G, Zikou A, Tsiouris $S$ et al. (2014) Comparison of diffusion tensor, dynamic susceptibility contrast MRI and 99mTcTetrofosmin brain SPECT for the detection of recurrent high grade glioma. Magn Reson Imaging, 32: 854-859.

16. Baek H, King H, Kim $N$ et al. (2012): Percent change of perfusion skewness and kurtosis: a potential imaging biomarker for early treatment response in patients with newly diagnosed glioblastomas. Radiology, 264: 834-843.
17. Chung W, Kim H, Kim N et al. (2013): Recurrent glioblastoma: optimum area under the curve method derived fromdynamic contrast-enhanced T1-weighted perfusion MR imaging. Radiology, 269: 561-568.

18. D'Souza M, Sharma R, Jaimini A et al. (2014): 11C-MET PET/CT and advanced MRI in the evaluation of tumor recurrence in high grade gliomas. Clin Nucl Med., 39: 791798.

19. Cha J, Kim S, Kim H et al. (2014): Differentiation of tumor progression from pseudo progression in patients with posttreatment glioblastoma using multiparametric histogram analysis. AJNR Am J Neuroradiol., 35: 1309-17.

20. Di Constanzo A, Scarabino T, Trojsi F et al. (2014): Recurrent glioblastoma multiforme versus radiation injury: a multiparametric 3-TMR approach. Radiol Med., 119: 616-624.

21. Sundgren $P$, Fan $X$, Weybright $P$ et al. (2006) Differentiation of recurrent brain tumor versus radiation injury using diffusion tensorimaging in patients with new contrast-enhancing lesions. Magn Reson Imaging, 24: 1131-1142. 